\title{
Nanoplastics and Arsenic Co-Exposures Exacerbate Oncogenic Biomarkers Under an in Vitro Long-Term Exposure Scenario
}

Ricard Marcos ( $\square$ ricard.marcos@uab.es )

Universitat Autonoma de Barcelona https://orcid.org/0000-0001-7891-357X

Irene Barguilla

Autonomous University of Barcelona: Universitat Autonoma de Barcelona

Josefa Domenech

Autonomous University of Barcelona: Universitat Autonoma de Barcelona

Laura Rubio

Pontificia Universidad Católica Madre y Maestra: Pontificia Universidad Catolica Madre y Maestra

Alba Hernández

Autonomous University of Barcelona Faculty of Biosciences: Universitat Autonoma de Barcelona

Facultat de Biociencies

\section{Research}

Keywords: polystyrene nanoplastics, arsenic, long-term co-exposure, cell transformation, genotoxic DNA damage, oncogenic phenotype, carcinogenesis.

Posted Date: November 11th, 2021

DOI: https://doi.org/10.21203/rs.3.rs-1042926/v1

License: (9) This work is licensed under a Creative Commons Attribution 4.0 International License.

Read Full License 


\section{Abstract \\ Background}

The increasing accumulation of plastic waste and the widespread presence of its derivatives, micro- and nanoplastics (MNPLs), call for an urgent evaluation of their potential health risks. In the environment, MNPLs coexist with other known hazardous contaminants and, thus, an interesting question arises as to whether MNPLs can act as carriers of such pollutants, modulating their uptake and their harmful effects. In this context, we have examined the interaction and joint effects of two relevant water contaminants: arsenic and polystyrene nanoplastics (PSNPLs), this last being a model of nanoplastic.

\section{Methods}

Since both agents are persistent pollutants, the potential effects have been evaluated under a chronic exposure scenario and measuring different effect biomarkers involved in the cell transformation process. Thus, cells previously transformed by chronic arsenic exposure were further exposed to PSNPLs, arsenic, and the combination PSNPLs/arsenic for 12 weeks.

\section{Results}

Interestingly, a physical interaction between both pollutants was demonstrated by using TEM/EDX methodologies. Results also indicate that the continuous co-exposure enhances the DNA damage and the aggressive features of the initially transformed phenotype. Remarkably, co-exposed cells present a higher proportion of spindle-like cells within the population, an increased capacity to grow independently of anchorage, as well as enhanced migrating and invading potentials when compared to cells exposed to arsenic or PSNPLs alone.

\section{Conclusions}

This study highlights the need for further studies exploring the long-term effects of contaminants of emerging concern such as MNPLs, and the importance of considering the behavior of mixtures as part of the hazard and human risk assessment approaches.

\section{Background}

The generalized utilization of single-use plastic in uncountable applications comes together with an alarming rise in the levels of plastic waste, which is becoming a pressing environmental issue. Under environmental conditions, plastic litter is subjected to fragmentation and degradation into the so-called micro- and nanoplastics (MNPLs) [1, 2]. While macroplastic pollution is the noticeable facet of this issue, the widespread presence of MNPLs in water, soil, and the air is equally worrisome. Indeed, it has recently 
been estimated that 8.3 million MNPLs particles contaminate each cubic meter of ocean water [3], and they are ubiquitously distributed on open sea and coasts [4]. Moreover, current research indicates that MNPLs' presence in freshwater and groundwater is as extensive as in marine systems $[5,6]$. Although there are still many questions to be answered on the potential hazard that MNPLs pollution poses to human health, recent studies evidence the MNPLs' potential to i) translocate through physiological barriers and internalize cells [7, 8]; ii) produce cytotoxicity [9]; iii) increase the levels of reactive oxygen species (ROS) [10]; iv) induce DNA damage [11]; and v) trigger the altered secretion of pro-inflammatory cytokines [12]. It is noteworthy the lack of exploration of MNPLs effects under long-term exposure conditions, which would be more descriptive of a real environmental exposure scenario.

In the actual context of environmental pollution, diverse contaminants coexist in the same site and, potentially, they can interact with each other. Therefore, increasing interest is being addressed towards the potential role of MNPLs as carriers for other contaminants. MNPLs' surface features give them the capacity to interact with and adsorb other compounds such as heavy metals [13], or organic pollutants $[14,15]$. Interestingly, cadmium, titanium, and lead have already been detected in MNPL samples collected on marine ecosystems [16], and traces of arsenic, titanium, nickel, and cadmium have been identified in polyethylene debris from the North Atlantic subtropical gyre [17]. Despite the potential risk derived from the role of MNPLs as carriers for other pollutants, the information on their combined effects is still very limited.

Arsenic is one of those metals potentially sharing environmental compartments with MNPLs. Inherently present in the Earth's crust, arsenic is part of geological formations widespread worldwide. The

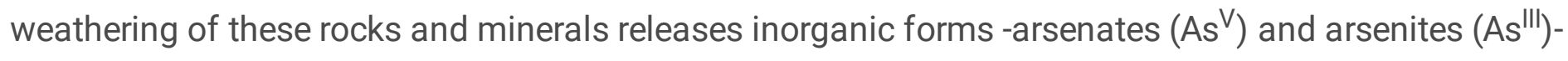
which enter the arsenic cycle as dust or dissolved in water, and are carried by rivers, rain, and groundwater $[18,19]$. Hence, the intake of contaminated water or food is the main route of exposure to this classic genotoxic and carcinogenic pollutant. Arsenic exposure affects large human populations that are at a higher risk to develop cardiovascular abnormalities, neurological alterations, hepatotoxicity, and most importantly cancer. Being a well-described carcinogen, long-term exposures to arsenic have been associated with lung, bladder, liver, skin, prostate, and kidney cancer [20]. Multiple studies have addressed arsenic-induced carcinogenesis following diverse in vitro, in vivo, and epidemiological approaches. In vitro, 12-30 weeks of chronic arsenic exposure transform very different cell models, including lung epithelial cells [21], prostate epithelial cells [22], breast epithelial cells [23], keratinocytes [24, 25], and fibroblasts [26].

As previously mentioned, the main health hazard associated with arsenic contamination is the intake of contaminated groundwater, which is also potentially contaminated with MNPLs. As a representative example, microplastic fibers have been detected in karstic groundwater systems at a concentration of 15.2 particles/L in certain areas of the USA [27]. There is still limited information regarding the quantification of MNPLs levels in groundwater, however given the widespread nature of plastic contamination, it seems likely that MNPLs and arsenic are found together in highly polluted areas. An important implication of this coexistence is that MNPLs could act as arsenic carriers, altering its 
toxicological profile. Further, considering the existing evidence of arsenic adsorption onto MNPLs [28, 29], it is very relevant to describe the probable health risk posed by this interaction, which remains unexplored up to date.

In this work, we have determined the interaction between arsenic and PSNPLs, the latter being one of the most abundant MNPLs in the environment [1]. Further, to assess the co-exposure impact under long-term conditions, we selected mouse embryonic fibroblasts previously transformed by 30 weeks of chronic exposure to sodium arsenite (As ${ }^{I I I}$ ) hereafter referred as arsenic-transformed cells (AsTC). The phenotypic and molecular changes that AsTC undergo during chronic arsenic exposure were well characterized by [30]. AsTC showed characteristic cancer-associated features such as morphological changes, differentiation status deregulation, and invasiveness potential [30]. Rather than evaluating tumor induction, the transformed status of our selected AsTC model allows us to examine the potential tumorpromoting effects of a MNPLs/As ${ }^{\text {III }}$ co-exposure, which might otherwise go unnoticed. Thus, aiming to assess whether the chronic PSNPLs exposure or the PSNPLs/As'II co-exposure exacerbate the arsenicinduced transformed phenotype, AsTC were further exposed for 12 weeks to PSNPLs, As ${ }^{\prime \prime \prime}$, or the combination of both compounds, and different hallmarks of carcinogenesis were evaluated.

\section{Methods}

\section{PSNPLs and y-PSNPLs characterization}

Pristine PSNPLs (PP-008-10) and yellow fluorophore-conjugated PSNPLs (y-PSNPLs) (FP-00552-2) were purchased from Spherotech (Chicago, USA). The pristine form of polystyrene (PS) particles was used for all the experiments carried out except for those in which the fluorescent marker was required, such as the visualization and the quantification of PS particles internalization by confocal microscopy and flow cytometry, respectively. PSNPLs used for the assays were characterized using Zetasizer and transmission electron microscopy (TEM) methodologies. To that purpose, the obtained dispersions were diluted to a final concentration of $100 \mu \mathrm{g} / \mathrm{mL}$ in distilled water. The hydrodynamic size and the Z-potential values for PSNPLs and y-PSNPLs dispersions were determined in a Malvern Zetasizer Nano ZS zen3600 device (Malvern, UK). All the parameters for each sample were measured in triplicates. TEM grids were dipped into PSNPLs and y-PSNPLs dispersions, and visualized on a JEOL JEM-1400 instrument (JEOL LTD, Tokyo, Japan). To determine the mean size, 100 randomly selected PSNPLs were measured using an Image $\mathrm{J}$ software with the Fiji extension.

\section{Cell culture conditions}

Mouse embryonic fibroblasts phenotypically sensitive to oxidative damage, and previously transformed by 30 weeks of continuous sodium arsenite (As $\left.{ }^{I I I}\right)$ exposure $(2 \mu \mathrm{M})$ [30], were used in this study and will be henceforth referenced as arsenic-transformed cells (AsTC). AsTC were grown in DMEM medium supplemented with fetal bovine serum, as previously reported [12].

\section{PSNPLs uptake by AsTC}


The cellular localization of y-PSNPLs was determined by confocal microscopy to assess PSNPLs internalization by AsTC. To this end, 80,000 AsTC were seeded in Glass Bottom Microwell dishes (MatTek, Ashland, USA) and exposed to 25 and $100 \mu \mathrm{g} / \mathrm{mL}$ y-PSNPLs, for $24 \mathrm{~h}$. The samples were washed with PBS 1X, and nuclei and cell membranes were stained with 1:500 Hoechst 33342 (ThermoFisher Scientific, Carlsbad, USA) and 1:500 Cellmask ${ }^{\mathrm{TM}}$ Deep Red plasma (ThermoFisher Scientific, Carlsbad, USA), respectively, for $15 \mathrm{~min}$ at room temperature. y-PSNPLs were detected thanks to the fluorophore to which they are conjugated. A Leica TCS SP5 confocal microscope was used to visualize two different randomly selected fields per sample, and images were processed with the software Image $\mathrm{J}$ with the Fiji extension. In addition, a quantification of the y-PSNPLs internalization was carried out by flow cytometry. Briefly, after the AsTC exposure to 25 and $100 \mu \mathrm{g} / \mathrm{mL}$ of y-PSNPLs, cells were washed with PBS 1X, trypsinized, centrifuged, and recovered in PBS $1 \mathrm{X}$ at a final concentration of $1 \times 10^{6}$ cells $/ \mathrm{mL}$ in FACS tubes. To select alive cells from the total population of the samples, 1:1000 propidium iodide was added before the analysis with a BD FACSCanto Flow Cytometer (BD Bioscience, Franklin Lakes, USA). About 10,000 living cells per sample were analyzed and the y-PSNPLs uptake was extrapolated from the mean fluorescence intensity of the living cells population. AsTC incubated with DMEM/F12 medium were used as a control in both assays.

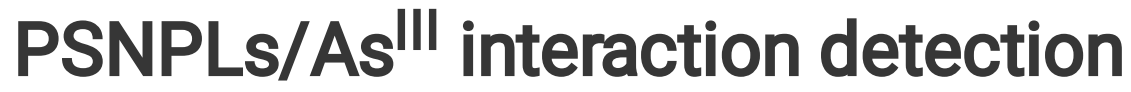

The interaction between As ${ }^{\text {III }}$ and PSNPLs was assessed by TEM. With this aim, the highest doses of both treatments were used. Concisely, a dilution in distilled water with a final concentration of $20 \mu \mathrm{M} A s^{\prime \prime \prime}$ and $100 \mu \mathrm{g} / \mathrm{mL}$ PSNPLs was incubated for $3 \mathrm{~h}$ at room temperature. TEM grids were dipped into the sample, and analyzed by transmission electron microscopy coupled with energy-dispersive X-ray spectroscopy (TEM-EDX). A TEM JEOL-2011 (200kV) instrument (JEOL LTD, Tokyo, Japan) was used to visualize the sample and take images, while an INCA detector (Oxford Instruments, United Kingdom) was used to determine the elementary composition of the sample to detect arsenic on PSNPLs surface.

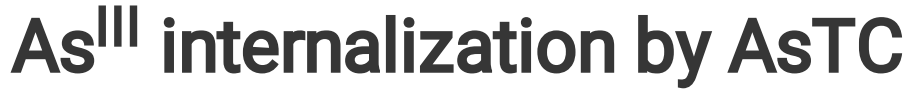

To evaluate and quantify the As ${ }^{\text {III }}$ uptake by AsTC, an analysis with inductively coupled plasma mass spectrometry (ICP-MS) was performed. AsTC were exposed to $20 \mu \mathrm{M} \mathrm{As}$ III combined with 0,25 , and 100 $\mu \mathrm{g} / \mathrm{mL}$ PSNPLs for $24 \mathrm{~h}$. AsTC non-exposed to As ${ }^{\prime \prime \prime}$, but exposed to 0,25 , and $100 \mu \mathrm{g} / \mathrm{mL}$ PSNPLs for 24 $\mathrm{h}$ were used as negative controls. After that, cells were washed with PBS 1X and trypsinized. Then, samples were centrifuged at 1,000 rpm for $8 \mathrm{~min}$, supernatants were discarded, and pellets were frozen at $-20{ }^{\circ} \mathrm{C}$ until 30 min digestion in concentrated $\mathrm{HNO}_{3}$ (Merck, supra pure) on a heat block at $105^{\circ} \mathrm{C}$. Finally, the amount of arsenic in each sample was determined using an ICP-MS 7500-ce device (Agilent Technologies, Santa Clara, USA).

\section{In vitro chronic PSNPLs and arsenic (co)exposure}

AsTC were (co)exposed for 12 weeks to $25 \mu \mathrm{g} / \mathrm{mL}$ PSNPLs, $2 \mu \mathrm{M}$ As ${ }^{\prime \prime \prime}$, or the combination of both treatments: $25 \mu \mathrm{g} / \mathrm{mL}$ PSNPLs/ $2 \mu \mathrm{M}$ As ${ }^{\text {III. }}$. These concentrations were selected being within the low range 
of doses with subtoxic effects. Replicates of exposed and passage-matched AsTC were maintained in two separate T-25 flasks, and grown under the culture conditions previously described. To ensure a constant exposure condition in the long-term exposure experiments, the cell culture medium was replaced every 2-3 days with new media containing the desired concentration of PSNPLs, As ${ }^{\text {III, }}$, f the combination PSNPLs/As"II.

\section{Comet assay}

The total and oxidative DNA damage (ODD) for AsTC under the different exposure scenarios was evaluated by the comet assay with/without FPG enzyme, as already described [31]. Sheet films of the type Gelbond® $(\mathrm{GF})$ were used as a support. Briefly, cells were collected by trypsinization, centrifuged, and resuspended in cold PBS at 17,500 cells $/ 25 \mu \mathrm{L}$. Then, cells were mixed with $0.75 \% \mathrm{LMP}$ agarose at $37^{\circ} \mathrm{C}(1: 10)$ and $7 \mu \mathrm{L}$ of the mixture was dropped onto the GF. The GF were lysed overnight, gently washed twice in enzyme buffer, and incubated in such buffer (negative control) or in FPG-containing enzyme buffer. After an electrophoresis buffer washing, GF were submitted to electrophoresis (20 min at $0.8 \mathrm{~V} / \mathrm{cm}$ and $300 \mathrm{~mA}$ ) at $4^{\circ} \mathrm{C}$. The GF, rinsed with cold PBS and fixed in absolute ethanol, were stained with SYBR Gold and analyzed according to their percentage of DNA in the tail, as an adequate measure of DNA damage, using a semi-automatic software scoring [11].

\section{Cell morphology and spindle-like-cell proportion calculation}

Morphological changes are associated with cell transformation and result from changes in the network of filaments constituting the cytoskeleton mainly responsible for controlling and maintaining cellular morphology and motility. To qualitatively evaluate cell morphology, cells were photographed with a Zeiss Observer A1 microscope. A quantification of the spindle-like cells was carried out with the software Image $\mathrm{J}$ with the Colocalization Object Counter plugin [32]. For 5 randomly selected images, total and spindlelike cells were counted and the proportion of spindle-like cells in each field was calculated.

\section{Soft-agar assay}

To assess the cells' anchorage-independent growth potential, colony formation in soft-agar was determined for AsTC and the different exposure conditions. The method recently described was used [33]. A suspension of 65,000 cells in $1.75 \mathrm{~mL}$ of DMEM containing $10 \%$ of FBS and $2.5 \mu \mathrm{g} / \mathrm{mL}$ Plasmocin was prepared and mixed with DMEM containing FBS, NEEA, L-Glu, penicillin-streptomycin, and bacto-agar. 20,000 cells $(1.5 \mathrm{~mL})$ were placed in each well of a 6 -well plate containing a $0.6 \%$ base agar. Plates were maintained at $37^{\circ} \mathrm{C}$ for 21 days. To detect cells able to form colonies they were stained with INT, scanned with a HP Scanjet G4050, and the resulting colonies were counted using the colony cell counter enumerator software OpenCFU (3.9.0).

\section{Invasion and migration assays}

The invasive potential of AsTC and those subjected to further As ${ }^{\text {III }}$ and PSNPLs exposures was evaluated by performing direct migration and invasion assays. To carry out the invasion assay, cells at $80 \%$ confluency were deprived of FBS for $24 \mathrm{~h}$. Deprived cells were placed on the apical side of a transwell 
insert cover with a Matrigel ${ }^{\circledR}$ mixture while the basolateral side contained DMEM complemented with FBS as chemoattractant medium. Cells were then allowed to invade for $48 \mathrm{~h}$ and invading cells were collected and counted. A modified version of the assay was performed to evaluate cell migration. The main steps were followed as described above; however, the cells were seeded on the top of the transwell without the Matrigel ${ }^{\circledR}$ coating.

\section{Tumorsphere formation assay}

AsTC and exposed cells were seeded at a density of 2,500 cells/mL on 96-well ultra-low-attachment plates (Corning, Costar-Corning, NY, USA) in serum-free DMEM/F12 supplemented with B27, $20 \mathrm{ng} / \mathrm{mL}$ basic fibroblast growth factor (bFGF) (both from Life Technologies, NY, USA), epithelial growth factor and $4 \mathrm{\mu g} / \mathrm{mL}$ heparin (both from Sigma-Aldrich, Germany). After 6 days of incubation $\left(5 \% \mathrm{CO}_{2}\right.$ and $37^{\circ} \mathrm{C}$ ), the tumorspheres were counted and photographed. Tumorspheres' size was assessed using ImageJ software.

\section{Results}

\section{PS materials characterization}

Both, PSNPLs and y-PSNPLs were visualized by TEM. As shown in Figure 1A, the commercial PSNPLs and y-PSNPLs dispersions consist of electrodense round-shaped particles. Particles' size was measured from TEM images by Image J, obtaining median sizes of $45.91 \mathrm{~nm}$ and $42.42 \mathrm{~nm}$ for PSNPLs and $\mathrm{y}$ PSNPLs, respectively (Figure 1B). PS materials were further characterized by Z-sizer and data obtained is summarized in Figure 1B. PSNPLs and y-PSNPLs hydrodynamic radius measured by DLS were larger than those measured from TEM images. Besides, polydispersity index ( $\mathrm{Pdl}$ ) values close to 0 indicate the samples are consistently monodispersions, and Z-potential measurements indicate high stability of the dispersions.

\section{Determination and quantification of y-PSNPLs uptake by AsTC}

The cellular location of y-PSNPLs after the internalization by AsTC was assessed by confocal microscopy. y-PSNPLs were found inside the cell cytoplasm in all conditions analyzed (Figure 2A). However, no difference in the nanoplastic internalization pattern could be deduced at first sight with the tested concentrations. To quantify y-PSNPLs cellular uptake, the mean fluorescence intensity of the living AsTC exposed to 25 and $100 \mu \mathrm{g} / \mathrm{mL}$ y-PSNPLs was determined by flow cytometry. As shown in Figure 2B, there is a dose-dependent significant increase of the fluorescence intensity, which reflects a greater internalization of y-PSNPLs as the selected doses increase.

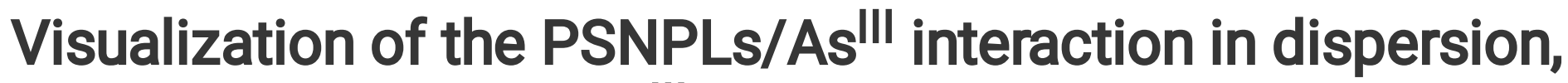 and quantification of As ${ }^{\text {III }}$ uptake by AsTC}


As ${ }^{\text {III }}$ and PSNPLs interactions in dispersion were visualized by TEM and the presence of arsenic was confirmed with the EDX analysis. As shown in Figure 3A, different types of associations between As ${ }^{\text {III }}$ and polystyrene particles were observed. On the one hand, arsenic was found associated with single PSNPLs. Differently, arsenic was also visualized forming aggregates that were, in turn, ringed by PSNPLs. The plot of the arsenic electrons transition shown in the diagrams obtained with EDX, confirmed that the electrodense shadow detected with TEM consists of As'"I. Once confirmed the interaction and the formation of PSNPLs/As"II complexes, arsenic uptake by AsTC was determined by ICP-MS. The amount of arsenic detected inside the cells did not show a PSNPLs dependency. As shown in Figure 3B, the amount of internalized arsenic remained unchangeable at the different PSNPLs concentrations assayed. The pg of arsenic measured were normalized to the number of cells in each sample. In the negative control samples, arsenic was under the limit of detection as expected. Since the ICP-MS device was unable to detect it, data are arbitrarily represented as $0.005 \mathrm{pg} \mathrm{As}$ 'III/cell.

\section{Both the co-exposure and the single pollutants exposure induce DNA damage}

Given the obtained evidence supporting the interaction between the selected pollutants, we evaluated the genotoxic potential of the chronic (co)exposure to arsenic and PSNPLs. The levels of DNA damage and oxidative DNA damage (ODD) of AsTC were assessed after 12-weeks of prolonged PSNPLs, As ${ }^{\prime \prime \prime}$, or PSNPLs/As ${ }^{\text {III }}$ exposure. As seen in Figure 4, all exposure scenarios tested led to an increase of the total (Figure 4A) and oxidative (Figure 4B) DNA damage, compared to the damage levels of unexposed AsTC. Interestingly, the levels of ODD were also significantly greater than those from PSNPLs-exposed AsTC. This indicates the potential induction of oxidative stress that would affect the DNA bases.

\section{The AsTC oncogenic phenotype is exacerbated by the long- term co-exposure to As ${ }^{\text {III }}$ and PSNPLs}

To determine whether the aggressiveness of the oncogenic phenotype of AsTC is enhanced after the 12week-extended exposure to As'"I, PSNPLs, or the combination PSNPLs/As'I", several carcinogenesis biomarkers were evaluated. The cells' proliferation rate remained unchanged during the weeks of longterm exposure (data not shown). However, as shown in Figure 5, a certain level of morphological changes is evidenced by the increase in the proportion of spindle-like cells in the culture of PSNPLs- and As IIIexposed cells, and in those subjected to the co-exposure, in comparison to non-exposed AsTC.

At a functional level, the soft-agar assay showed a significant 6-fold increase in the number of colonies formed by AsTC under PSNPLs/As"ll exposure settings, when compared with passage-matched or singleexposed AsTC (Figure 6A). Interestingly, no effects of the chronic PSNPLs exposure were observed. Regarding the cells' migrating (Figure 6B) and invading (Figure 6C) potential, PSNP-and As'll-exposed cells showed a similar ability to cross the porous membrane and translocate to the basolateral part of the transwell as that of AsTC, while the number of PSNPLs/As ${ }^{\text {III }}$ co-exposed cells able to migrate and invade, was 2 -fold and 3-fold higher, respectively. 


\section{The increased aggressiveness of AsTC's oncogenic phenotype is not related to an increasing stem-like cells population}

The cells' capacity to grow as tumorspheres is associated with the presence of stem or progenitor cells in tumor cell populations. Thus, the number of tumorspheres formed can be used to characterize the cancer stem-like cells within a population of in vitro cultured cancer cells resulting from the applied exposures. When we evaluated if the potential of AsTC to form tumorspheres was exacerbated by the extended PSNPLs, As"II, and combined exposures, no differences were found (Figure 7). This would indicate that, under the tested exposure conditions, the pollutants do not induce an increase the number of cancer stem cells within the AsTC population.

\section{Discussion}

The widespread and ever-rising amount of plastic litter is closely linked to the increasing levels of MNPLs found in all environmental compartments. These tiny particles $(<5 \mathrm{~mm})$ are ubiquitously distributed in soil, air, freshwater, and marine ecosystems from where they easily enter the trophic chain [34]. As a result, humans are believed to be mainly exposed to MNPLs via the ingestion of contaminated food or water, but also through other routes such as inhalation or dermal deposition [35]. Under this potential broad human exposure scenario, urgent hazard assessment is required.

Great efforts are being addressed to the understanding of the environmental and ecotoxicological effects of these emergent contaminants. However, studies based on mammal and human models focusing on the characterization of the MNPLs' impact on human health are still limited [36]. Among those available in the literature, it has been fairly described that MNPLs significantly internalize cells and translocate through physiological barriers [7, 8]; however, whether this uptake results in a biological impact is not sufficiently clear. While some authors describe a lack of cytotoxic and cytostatic effects [37,38], others have reported MNPL-induced ROS production and pro-inflammatory responses in vitro, as well as mild histological lesions and metabolic disorders in rodent systems [39]. Regardless of these disparities, overall, MNPLs are considered to have low acute toxicity. Nonetheless, much remains to be unveiled in terms of MNPLs' long-term effects and their role as carriers of different environmental pollutants, which is now attracting attention as a potential toxicological risk associated with MNPLs exposure.

In this context, our work contributes to the field in two ways: (1) by the analysis of the impact of MNPLs and arsenic (co)exposures under a long-term exposure scenario; and (2) by the establishment of a model in which the already damaged genetic background of cells may render them more susceptible to the alterations induced by MNPLs, allowing the detection of typically unnoticed mild effects.

Our selected co-contaminants of study are As ${ }^{\text {III }}$ and PSNPLs, as representative legacy and emergent contaminants, respectively. They share a ubiquitous environmental distribution although both have major 
implications in terms of human exposure via the intake of contaminated water and, at a lesser proportion, inhalation. As a result, primary target organs (gastrointestinal and respiratory tracts) are potentially affected by both contaminants $[40,41]$. Thus, these coexisting contaminants could have a joint impact on human health. Indeed, there is accumulating evidence hinting at MNPLs/arsenic interaction. Arsenic adsorption onto MNPLs has already been reported in debris samples collected from the open sea [17]. Besides, laboratory studies have confirmed arsenic adsorption onto polystyrene microplastic particles [29], and polytetrafluoroethylene microparticles [28]. Accordingly, our data demonstrate that arsenic adsorbs onto single PS particles, and it can form PSNPLs/As ${ }^{\text {III }}$ aggregates (see Figure $3 \mathrm{~A}$ ). Although the proportion of interactions within our samples are not quantifiable with the use of TEM/EDX, the physical interaction is observed and, thus, the generation of a certain number of PSNPLs/As ${ }^{\text {III }}$ complexes could induce differential effects compared to those of the addition of the arsenic and MNPLs as independent compounds.

Aiming to test the long-term impact of the PSNPLs/As ${ }^{\text {III }}$ complexes, and to demonstrate whether the effects induced by arsenic exposure are exacerbated under a co-exposure scenario, we have evaluated endpoints regarding genotoxicity and carcinogenicity after the chronic (co)exposure of AsTC, our selected in vitro model. The used AsTC derive from MEF cells previously demonstrated to be sensitive to oxidative stress which is closely linked with genotoxicity, genomic and chromosomal instability, and the eventual transformation driven by 30-weeks of chronic arsenic exposure [30, 31]. We assume the compromised genetic background of these cells can be helpful to make more evident the biological impact of MNPLs and their co-exposures.

The high cellular uptake of PSNPLs in our system (see Figures 2A and 2B), led us to consider whether the PSNPLs/As ${ }^{\text {III }}$ interaction would translate into an increased arsenic bioavailability and a higher internalization rate. This phenomenon has already been described upon in vitro co-exposures to arsenic and nanoparticles (NPs) such as $\mathrm{TiO}_{2} \mathrm{NPs}$ and $\mathrm{SiO}_{2} \mathrm{NPs}[42,43]$, and after the combined exposure to PSNPLs and AgNPs [44]. However, as shown in Figure 3B, the levels of arsenic internalized in AsTC remained stable with increasing doses of PSNPLs. Therefore, the remarkable genotoxic/oncogenic effects observed upon PSNPLs/As ${ }^{\text {III }}$ co-exposure are not due to the PSNPL-mediated facilitation of As ${ }^{\text {III }}$ uptake, but rather due to potential alterations induced by PSNPLs/As ${ }^{\text {III }}$ at the molecular level.

Among those notable effects of arsenic and MNPLs (co)exposure in our system, we have found a significant induction of both total and oxidative DNA damage. Arsenic is a well-known genotoxic compound and one of its most studied mechanisms of action is the induction of ROS and oxidative stress [45]. In addition, plenty of those studies reporting adverse effects of MNPLs have detected increased ROS levels and DNA damage after short-term exposures [10-12, 46]. Concordantly, with the analysis of the long-term effects of the exposure to PSNPLs, As ${ }^{\text {III }}$, and PSNPLs/As ${ }^{\text {III }}$ we found a significant increase in the total and oxidative DNA damage when compared with passage-matched AsTC (see Figure 4). Interestingly, the oxidative damage derived from the PSNPLs/As ${ }^{\text {III }}$ co-exposure is significantly higher than that observed after single exposures (see Figure 4B). This effect of contaminant 
mixtures enhancing arsenic-induced oxidative stress and genotoxicity has also been recently reported after short-term exposures when analyzing the impact of the co-exposure to $\mathrm{As}^{\mathrm{III}} / \mathrm{TiO}_{2} \mathrm{NPs}$ [42], $\mathrm{As}^{\mathrm{III} /} / \mathrm{SiO}_{2} \mathrm{NPs}$ [43], and As'l'/polystyrene microplastics [47]. Therefore, our results and those from other groups contribute to support the existence of positive interactions between contaminants.

Remarkably, the positive interaction between As ${ }^{\text {III }}$ and PSNPLs also adds to the aggressiveness of the arsenic-induced oncogenic phenotype. Arsenic capacity to drive in vitro carcinogenicity is well established [48], while this potential aspect of MNPLs' long-term impact has not been explored up to date. In in vitro studies, the usefulness of a battery of cancer hallmarks to assess the transformed status of cells has been proposed [49]. These include morphological changes, accelerated proliferation, secretome alterations, metastatic potential, and deregulation of the differentiation status. The measure of these features has been proven useful to assess arsenic-induced carcinogenesis before [50-52]. In the present study passage-matched AsTC that, as previously mentioned meet all endpoints, display an evident transformed phenotype [30]. Interestingly, according to our data, these hallmarks remain unchanged for AsTC subjected to 12 weeks of PSNPLs or As ${ }^{\text {III }}$ single exposure but are significantly enhanced under PSNPLs/As ${ }^{\text {III }}$ co-exposure settings. The increment on the proportion of spindle-like cells within the population (see Figure $5 \mathrm{~A}$ and $\mathrm{B}$ ), and especially the dramatically increased capacity of cells to grow independently of anchorage (see Figure 6A), migrate (see Figure 6B), and invade (see Figure 6C) confirm the co-exposure-mediated acquisition of a further aggressive transformed phenotype. To better characterize the oncogenic features of the (co)exposed AsTC, we evaluated their tumorsphere-forming ability as a marker of the stemness status in our cell population. Different studies have linked the conversion of non-stem cells to cancer stem cells with carcinogenesis [53], and, specifically, with arsenicinduced carcinogenesis [54-57]. However, we did not find significant differences under the different exposure scenarios tested, thus stemness induction seems not to be the mechanism by which PSNPLs/As' ${ }^{\text {III }}$ promotes tumor aggressiveness. Taken together, these findings highlight the urgent need to explore MNPLs' long-term effects and their potential role as co-carcinogens with other environmental pollutants.

\section{Conclusions}

As a summary, in the present work, we have demonstrated that the long-term concurrent exposure to subtoxic doses of arsenic and PSNPLs significantly enhances the arsenic-associated transformed phenotype and genotoxicity. Further, we have shown that PSNPLs and arsenic physically interact. However, this interaction is not associated with an increased arsenic bioavailability and, therefore, the mechanism by which PSNPLs add to arsenic's impact requires further research. Importantly, while the evaluation of the effects induced by a single exposure to MNPLs is still of great relevance, it is also necessary to explore whether new differential effects arise with environmentally relevant complex mixtures. Indeed, our results support considering co-exposure scenarios as an essential part of the emergent pollutants' hazard assessment. 


\section{Declarations}

\section{Acknowledgements}

We wish to thank Mr. C. Valiente for his technical assistance. The authors I. Barguilla and J. Domenech were supported by PhD fellowships from the Universitat Autònoma de Barcelona [PIF-UAB]. L. Rubio was supported by the Fondo Nacional de Innovación y Desarrollo Científico y Tecnológico (FONDOCYT) República Dominicana (Project 2018-2019-2B2-093).

\section{Funding}

This project has received funding from the European Union's Horizon 2020 research and innovation programme under grant agreement No 965196.

\section{Availability of data and materials}

The data supporting our conclusions is included in the main body of the manuscript.

\section{Authors' contributions}

$\mathrm{RM}$ and $\mathrm{AH}$ planned the experiments. IB, $\mathrm{LR}$, and JD carried out the experimental part. IB analyzed the data, carried out the statistical analysis, and prepared tables/figures. IB, RM, and AH wrote the final manuscript. All authors reviewed and approved the final manuscript.

\section{Ethics approval and consent to participate}

Not applicable.

\section{Consent for publication}

Not applicable.

\section{Competing interests}

The authors declared that they have no competing interests

\section{References}

1. Bouwmeester $\mathrm{H}$, Hollman $\mathrm{PCH}$, Peters RJB. Potential health impact of environmentally released micro- and nanoplastics in the human food production chain: Experiences from Nanotoxicology. Environ Sci Technol. 2015;49:8932-8947.

2. EFSA. Presence of microplastics and nanoplastics in food with particular focus on seafood. EFSA J. 2016;14:1-30. 
3. Brandon JA, Freibott A, Sala LM. Patterns of suspended and salp-ingested microplastic debris in the North Pacific investigated with epifluorescence microscopy. Limn Ocean Lett. 2020;5:46-53.

4. Li WC, Tse HF, Fok L. Plastic waste in the marine environment: A review of sources occurrence and effects. Sci Total Environ. 2016;566-567:333-349.

5. Eerkes-Medrano D, Thompson RC, Aldridge DC. Microplastics in freshwater systems: a review of the emerging threats identification of knowledge gaps and prioritisation of research needs. Water Res. 2015;75:63-82.

6. Wanner P. Plastic in agricultural soils -A global risk for groundwater systems and drinking water supplies? -A review. Chemosphere 2021;264:128453.

7. Hesler M, Aengenheister L, Ellinger B, Drexel R, Straskraba S, Jost C, Wagner S, Meier F, von Briesen $H$, Büchel C, Wick P, Buerki-Thurnherr T, Kohl Y. Multi-endpoint toxicological assessment of polystyrene nano- and microparticles in different biological models in vitro. Toxicol in Vitro 2019;61:104610.

8. Domenech J, Hernández A, Rubio L, Marcos R, Cortés C. Interactions of polystyrene nanoplastics with in vitro models of the human intestinal barrier. Arch Toxicol. 2020;94:2997-3012.

9. Xu M, Halimu G, Zhang Q, Song Y, Fu X, Li Y, Li Y, Zhang H. Internalization and toxicity: A preliminary study of effects of nanoplastic particles on human lung epithelial cell. Sci Total Environ. 2019;694:133794.

10. Liu Z, Huang Y, Jiao Y, Chen Q, Wu D, Yu P, Li Y, Cai M, Zhao Y. Polystyrene nanoplastic induces ROS production and affects the MAPK-HIF-1/NFkB-mediated antioxidant system in Daphnia pulex. Aquat Toxicol. 2020;220:105420.

11. Rubio L, Barguilla I, Domenech J, Marcos R, Hernández A. Biological effects including oxidative stress and genotoxic damage of polystyrene nanoparticles in different human hematopoietic cell lines. J Hazard Mater. 2020;398:122900.

12. Ballesteros S, Domenech J, Barguilla I, Cortés C, Marcos R, Hernández A. Genotoxic and immunomodulatory effects in human white blood cells after ex vivo exposure to polystyrene nanoplastics. Environ Sci Nano. 2020;7:3431-3446.

13. Godoy V, Blázquez G, Calero M, Quesada L, Martín-Lara MA. The potential of microplastics as carriers of metals. Environ Pollut. 2019:255:113363.

14. Hüffer T, Hofmann T. Sorption of non-polar organic compounds by micro-sized plastic particles in aqueous solution. Environ Pollut. 2016;214:194-201.

15. Lin W, Jiang R, Wu J, Wei S, Yin L, Xiao X, Hu S, Shen Y, Ouyang G. Sorption properties of hydrophobic organic chemicals to micro-sized polystyrene particles. Sci Total Environ. 2019;690:565572.

16. Massos A, Turner A. Cadmium lead and bromine in beached microplastics. Environ Pollut. 2017;227:139-145.

17. Prunier J, Maurice L, Perez E, Gigault J, Pierson Wickmann AC, Davranche M, Ter Halle A. Trace metals in polyethylene debris from the North Atlantic subtropical gyre. Environ Pollut. 2019;245:371379. 
18. Smedley PL, Kinniburgh DG. A review of the source behaviour and distribution of arsenic in natural waters. Appl Geochem. 2002;17:517-568.

19. Ravenscroft P, Brammer H, Richards K. Arsenic pollution. In Arsenic Pollution: A Global Synthesis. Wiley-Blackwell. A John Wiley and Sons Ltd. 2009;pp:616.

20. IARC. Arsenic metals fibres and dusts. Monographs on the evaluation of carcinogenic risk to humans. Int Agency Res Cancer. 2012;100:407-443.

21. Stueckle TA, Lu Y, Davis ME, Wang L, Jiang BH, Holaskova I, Schafer R, Barnett JB, Rojanasakul Y. Chronic occupational exposure to arsenic induces carcinogenic gene signaling networks and neoplastic transformation in human lung epithelial cells. Toxicol Appl Pharmacol. 2012;61:204-216.

22. Treas J, Tyagi T, Singh KP. Chronic exposure to arsenic estrogen and their combination causes increased growth and transformation in human prostate epithelial cells potentially by hypermethylation-mediated silencing of MLH1. Prostate 2013;73:1660-1672.

23. Xu Y, Tokar EJ, Waalkes MP. Arsenic-induced cancer cell phenotype in human breast epithelia is estrogen receptor-independent but involves aromatase activation. Arch Toxicol. 2014;88:263-274.

24. Pi J, Diwan BA, Sun Y, Liu J, Qu W, He Y, Styblo M, Waalkes MP. Arsenic-induced malignant transformation of human keratinocytes: Involvement of Nrf2. Free Radic Biol Med. 2008;45:651-658.

25. Li Y, Ling M, Xu Y, Wang S, Li Z, Zhou J, Wang X, Liu Q. The repressive effect of NF-kB on p53 by mot2 is involved in human keratinocyte transformation induced by low levels of arsenite. Toxicol Sci. 2010;116:174-182.

26. Shearer MJJ, Wold EA, Umbaugh CS, Lichti CF, Nilsson CL, Figueiredo ML. Inorganic arsenic-related changes in the stromal tumor microenvironment in a prostate cancer cell-conditioned media. Environ Health Perspect. 2016;124:1009-1015.

27. Panno SV, Kelly WR, Scott J, Zheng W, McNeish RE, Holm N, Hoellein TJ, Baranski EL. Microplastic contamination in karst groundwater systems. Ground Water 2019;57:189-196.

28. Dong Y, Gao M, Song Z, Qiu W. Adsorption mechanism of As(III) on polytetrafluoroethylene particles of different size. Environ Pollut. 2019;254:112950.

29. Dong Y, Gao M, Song Z, Qiu W. As(III) adsorption onto different-sized polystyrene microplastic particles and its mechanism. Chemosphere 2020;239:124792.

30. Bach J, Peremartí J, Annangi B, Marcos R, Hernández A. Oxidative DNA damage enhances the carcinogenic potential of in vitro chronic arsenic exposures. Arch Toxicol. 2016;90:1893-1905.

31. Bach J, Sampayo-Reyes A, Marcos R, Hernández A. Ogg1 genetic background determines the genotoxic potential of environmentally relevant arsenic exposures. Arch Toxicol. 2014;88:585-596.

32. Lunde A, Glover JC. A versatile toolbox for semi-automatic cell-by-cell object-based colocalization analysis. Sci Rep. 2020;10:19027.

33. Ballesteros S, Domenech J, Velázquez A, Marcos R, Hernández A. Ex vivo exposure to different types of graphene-based nanomaterials consistently alters human blood secretome. J Hazard Mater. 2021;414:125471. 
34. Correia-Prata J, da Costa JP, Lopes I, Duarte AC, Rocha-Santos T. Environmental exposure to microplastics: An overview on possible human health effects. Sci Total Environ. 2020;702:134455.

35. Lehner R, Weder C, Petri-Fink A, Rothen-Rutishauser B. Emergence of nanoplastic in the environment and possible impact on human health. Environ Sci Technol. 2019;53:1748-1765.

36. Toussaint B, Raffael B, Angers-Loustau A, Gilliland D, Kestens V, Petrillo M, Rio-Echevarria IM, Van den Eede G. Review of micro- and nanoplastic contamination in the food chain. Food Addit Contam. Part A Chem Anal Control Expo Risk Assess. 2019;36:639-673.

37. Stock V, Böhmert L, Lisicki E, Block R, Cara-Carmona J, Pack LK, Selb R, Lichtenstein D, Voss L, Henderson CJ, Zabinsky E, Sieg H, Braeuning A, Lampen A. Uptake and effects of orally ingested polystyrene microplastic particles in vitro and in vivo. Arch Toxicol. 2019;93:1817-1833.

38. Cortés C, Domenech J, Salazar M, Pastor S, Marcos R, Hernández A. Nanoplastics as a potential environmental health factor: Effects of polystyrene nanoparticles on human intestinal epithelial Caco-2 cells. Environ Sci Nano 2020;7:272-285.

39. Yong CQY, Valiyaveetill S, Tang BL. Toxicity of microplastics and nanoplastics in Mammalian systems. Int J Environ Res Public Health 2020;17:1509.

40. Stapleton PA. Toxicological considerations of nano-sized plastics. AIMS Environ Sci. 2019:6:367378.

41. Palma-Lara I, Martínez-Castillo M, Quintana-Pérez JC, Arellano-Mendoza MG, Tamay-Cach F, Valenzuela-Limón OL, García-Montalvo EA, Hernández-Zavala A. Arsenic exposure: A public health problem leading to several cancers. Reg Toxicol Pharmacol. 2020;110:104539.

42. Wang X, Liu Y, Wang J, Nie Y, Chen S, Hei TK, Deng Z, Wu L, Zhao G, Xu A. Amplification of arsenic genotoxicity by $\mathrm{TiO}_{2}$ nanoparticles in mammalian cells: new insights from physicochemical interactions and mitochondria. Nanotoxicology 2017:11:978-995.

43. Ahamed M, Akhtar MJ, Alhadlaq HA. Co-exposure to $\mathrm{SiO}_{2}$ nanoparticles and arsenic induced augmentation of oxidative stress and mitochondria-dependent apoptosis in human cells. Int J Environ Res Publ Health 2019;16:3199.

44. Domenech J, Cortés C, Vela L, Marcos R, Hernández A. Polystyrene nanoplastics as carriers of metals. Interactions of polystyrene nanoparticles with silver nanoparticles and silver nitrate and its effects on human intestinal Caco-2 cells. Biomolecules 2021;11:859.

45. Jomova K, Jenisova Z, Feszterova M, Baros S, Liska J, Hudecova D, Rhodes CJ, Valko M. Arsenic: Toxicity oxidative stress and human disease. J Appl Toxicol. 2011;31:95-107.

46. Schirinzi GF, Pérez-Pomeda I, Sanchís J, Rossini C, Farré M, Barceló D. Cytotoxic effects of commonly used nanomaterials and microplastics on cerebral and epithelial human cells. Environ Res. 2017;159:579-587.

47. Wu B, Wu X, Liu S, Wang Z, Chen L. Size-dependent effects of polystyrene microplastics on cytotoxicity and efflux pump inhibition in human Caco-2 cells. Chemosphere 2019;221:333-341. 
48. Zhou Q, Xi S. A review on arsenic carcinogenesis: Epidemiology metabolism genotoxicity and epigenetic changes. Reg Toxicol Pharmacol. 2018;99:78-88.

49. Hanahan D, Weinberg RA. Hallmarks of cancer: The next generation. Cell 2011;144:646-674.

50. Tokar EJ, Diwan BA, Waalkes MP. Arsenic exposure transforms human epithelial stem/ progenitor cells into a cancer stem-like phenotype. Environ Health Perspect. 2010;118:108-115.

51. Person RJ, Olive Ngalame NN, Makia NL, Bell MW, Waalkes MP, Tokar EJ. Chronic inorganic arsenic exposure in vitro induces a cancer cell phenotype in human peripheral lung epithelial cells. Toxicol Appl Pharmacol. 2015;286:36-43.

52. Ganapathy S, Liu J, Xiong R, Yu T, Makriyannis A, Chen C. Chronic low dose arsenic exposure preferentially perturbs mitotic phase of the cell cycle. Genes Cancer 2019;10:39-51.

53. Afify SM, Seno M. Conversion of stem cells to cancer stem cells: Undercurrent of cancer initiation. Cancers 2019;11:1-19.

54. Ngalame NNO, Luz AL, Makia N, Tokar EJ. Arsenic alters exosome quantity and cargo to mediate stem cell recruitment into a cancer stem cell-like phenotype. Toxicol Sci. 2018;165:40-49.

55. Ooki A, Begum A, Marchionni L, Vandenbussche CJ, Mao S, Kates M, Hoque MO. Arsenic promotes the COX2/PGE2-SOX2 axis to increase the malignant stemness properties of urothelial cells. Int $\mathrm{J}$ Cancer 2018;143:113-126.

56. Xiao T, Xue J, Shi M, Chen C, Luo F, Xu H, Chen X, Sun B, Sun Q, Yang Q, Dai X, Zhang A, Tang H, Liu Q. Circ008913 via miR-889 regulation of DAB2IP/ZEB1 is involved in the arsenite-induced acquisition of CSC-like properties by human keratinocytes in carcinogenesis. Metallomics 2018;10:1328-1338.

57. Wang Z, Yang P, Xie J, Lin HP, Kumagai K, Harkema J, Yang C. Arsenic and benzo[a]pyrene coexposure acts synergistically in inducing cancer stem cell-like property and tumorigenesis by epigenetically down-regulating SOCS3 expression. Environ Int. 2020;137:105560.

\section{Figures}


A
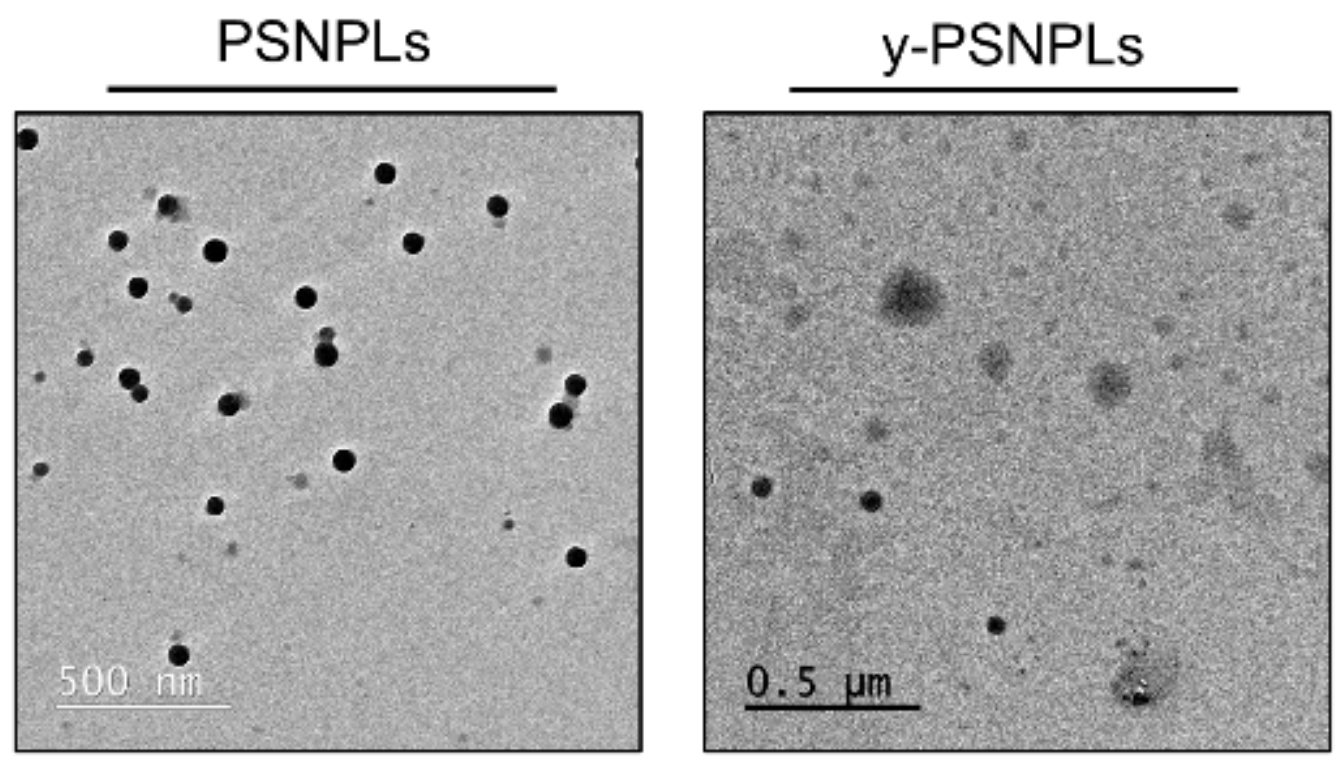

B

PSNPLs

Median size $(\mathrm{nm})$ (TEM)

Size (nm) (DLS)

Pdl (DLS)

Z-potential (mV) (LDV)

Mobility $(\mu \mathrm{m} \mathrm{cm} / \mathrm{Vs})(\mathrm{LDV})$
45.91

$86.33 \pm 10.20 \quad 112.87 \pm 3.11$

$0.10 \pm 0.09$

$-36.00 \pm 7.88 \quad-45.97 \pm 3.84$

$-2.29 \pm 0.10$

$-3.76 \pm 0.38$

Figure 1

PS nanomaterials characterization. (A) Representative TEM images of PSNPLs and y-PSNPL. (B) PSNPLs and y-PSNPLs characterization by TEM (median size calculated measuring 100 randomly selected PSNPLs) and Zetasizer Nano ZS (mean \pm SD). $100 \mu \mathrm{g} / \mathrm{mL}$ dilutions in distilled water of each material were used for the visualization and characterization. 
A
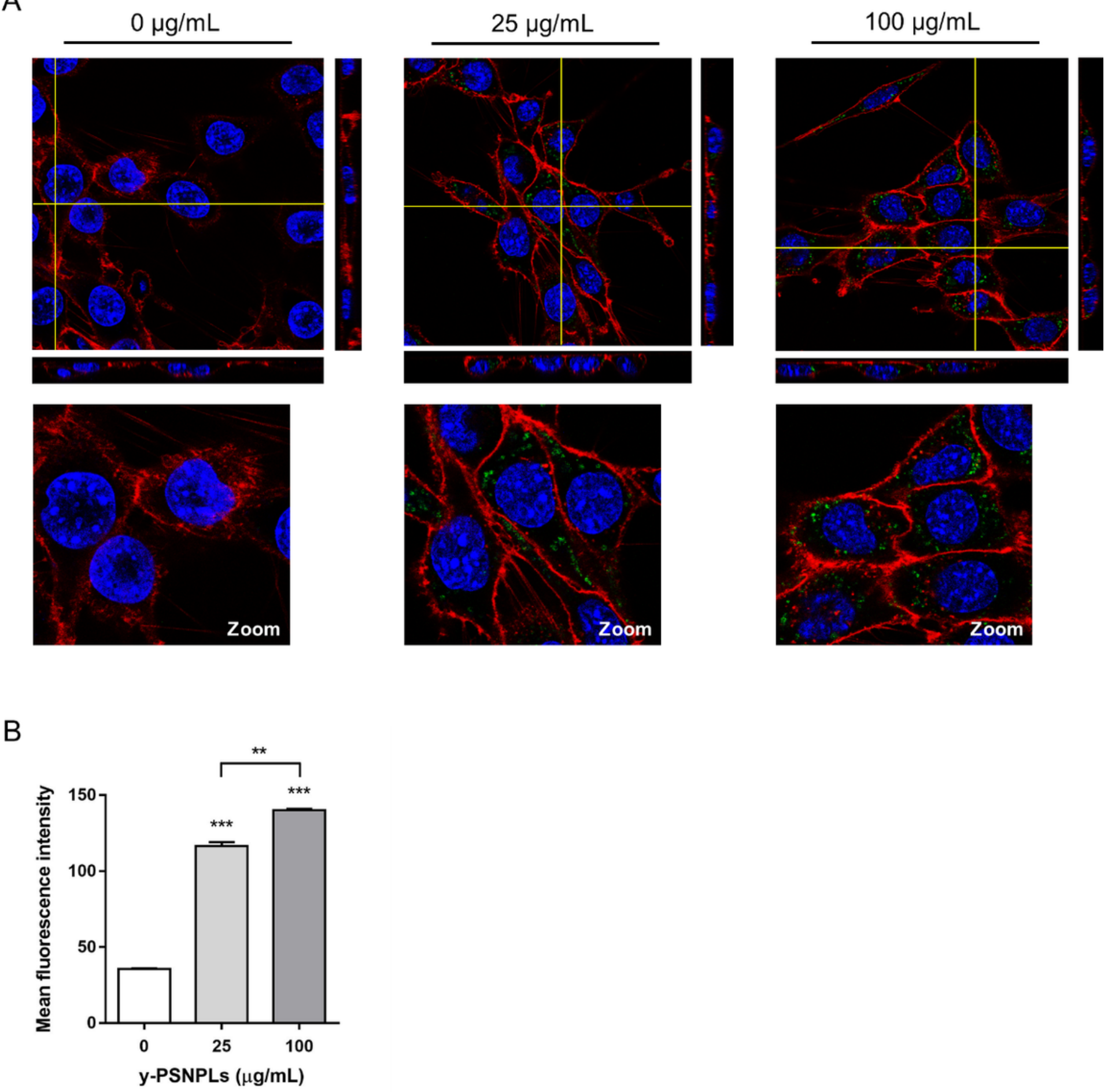

Figure 2

y-PSNPLs uptake by AsTC. (A) Images of the AsTC taken with confocal microscopy after the exposure to 0,25 , and $100 \mu \mathrm{g} / \mathrm{mL}$ y-PSNPLs for $24 \mathrm{~h}$. Nuclei (showed in blue) were stained with Hoechst and cell membranes (showed in red) were stained with CellMask. y-PSNPLs are shown in green. Yellow lines point out the plane from where orthogonal views are projected. (B) y-PSNPLs intake by AsTC after $24 \mathrm{~h}$ of exposure to 0,25 , and $100 \mu \mathrm{g} / \mathrm{mL}$. The mean fluorescence intensity of the living AsTC total population is 
represented. Data are shown as mean \pm SEM and analyzed by the Student's t-test ( ${ }^{\star \star *} \mathrm{P}<0.001$, ${ }^{\star *} \mathrm{P}<$ 0.01, compared to non-exposed AsTC controls or other conditions as indicated).

A
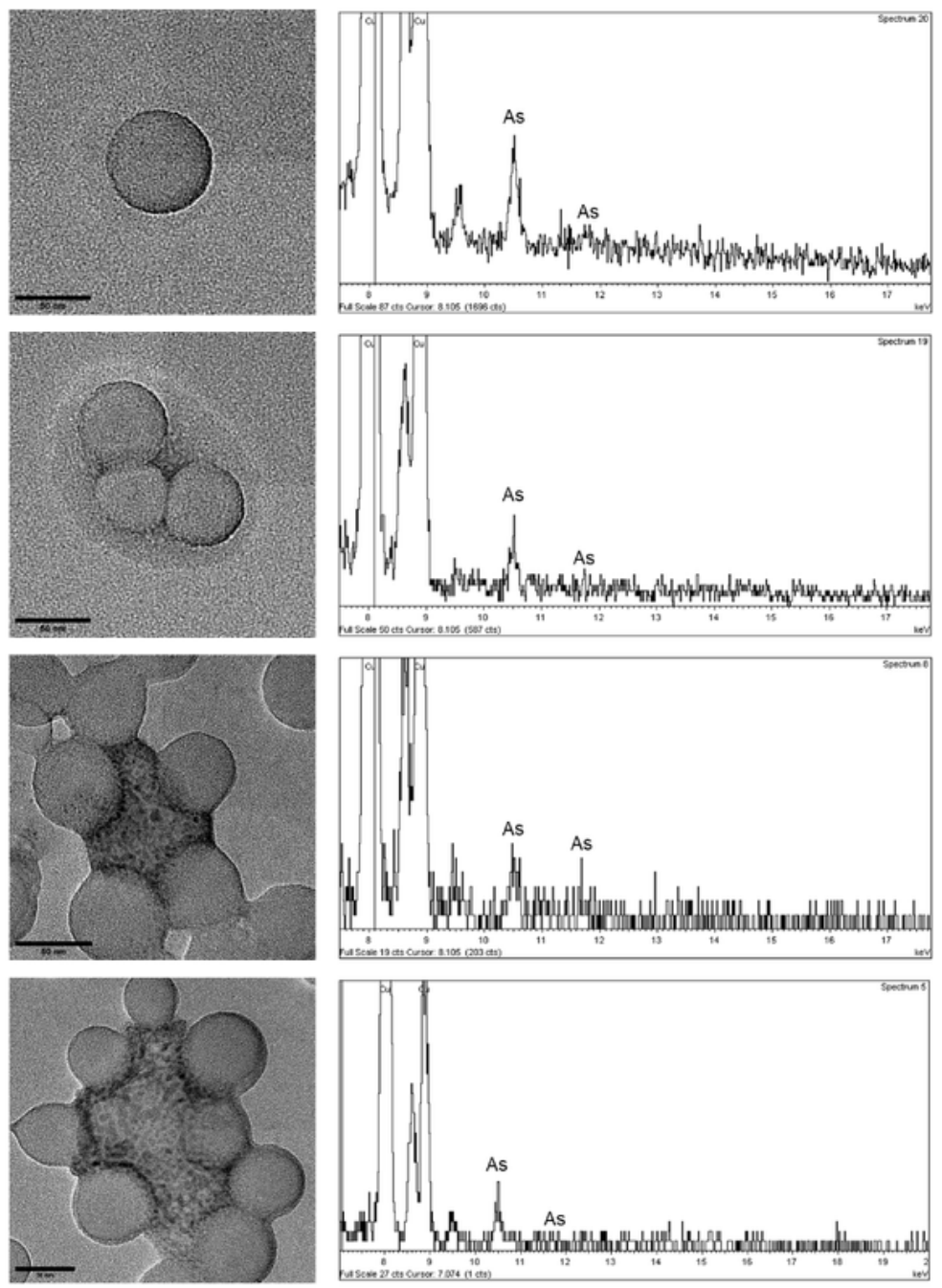

B

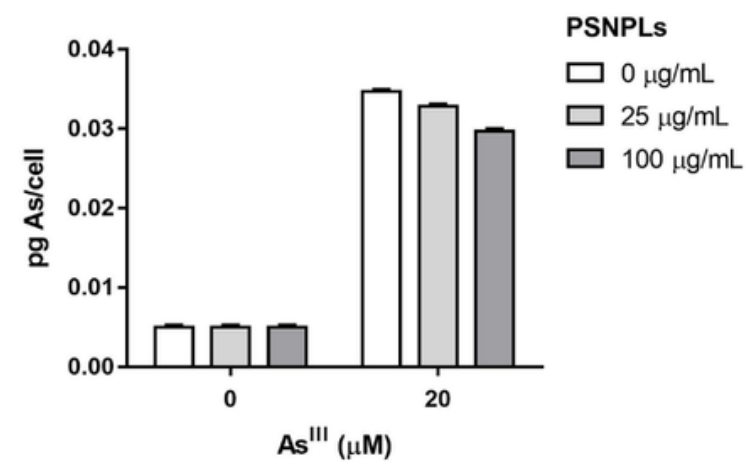

Figure 3

Determination of the interaction between PSNPLs and AsIII, and the AsIll uptake by AsTC. (A) Representative TEM images with their respective energy-dispersive X-ray EDX spectra indicating the chemical elemental characterization. (B) Quantification of the arsenic internalization by AsTC. Arsenic 
was not detected in control samples; thus, results are arbitrarily represented as $0.005 \mathrm{pg}$ of AsIll. Data are represented as mean \pm SEM and was analyzed by the Student's t-test.

A

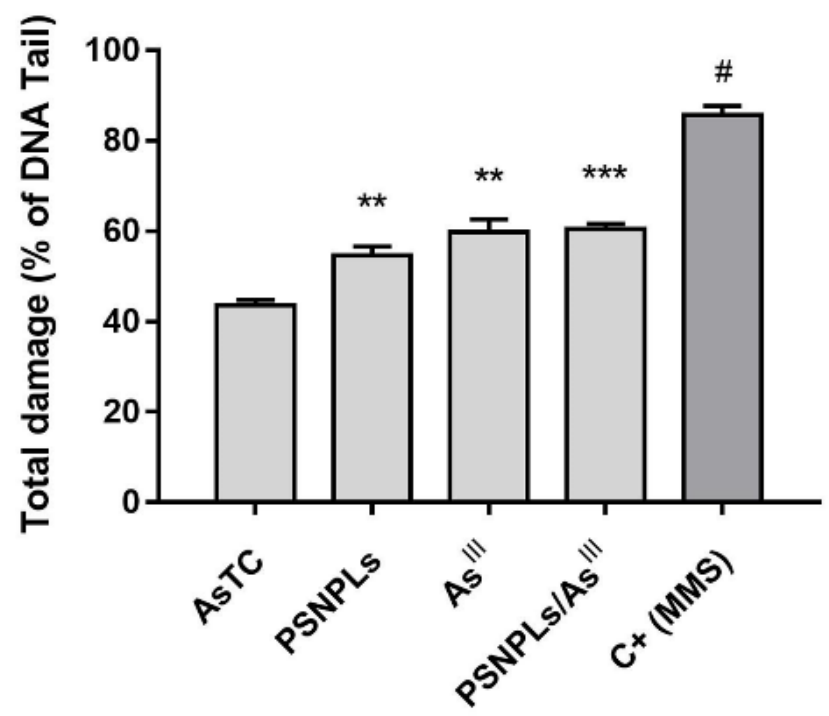

B

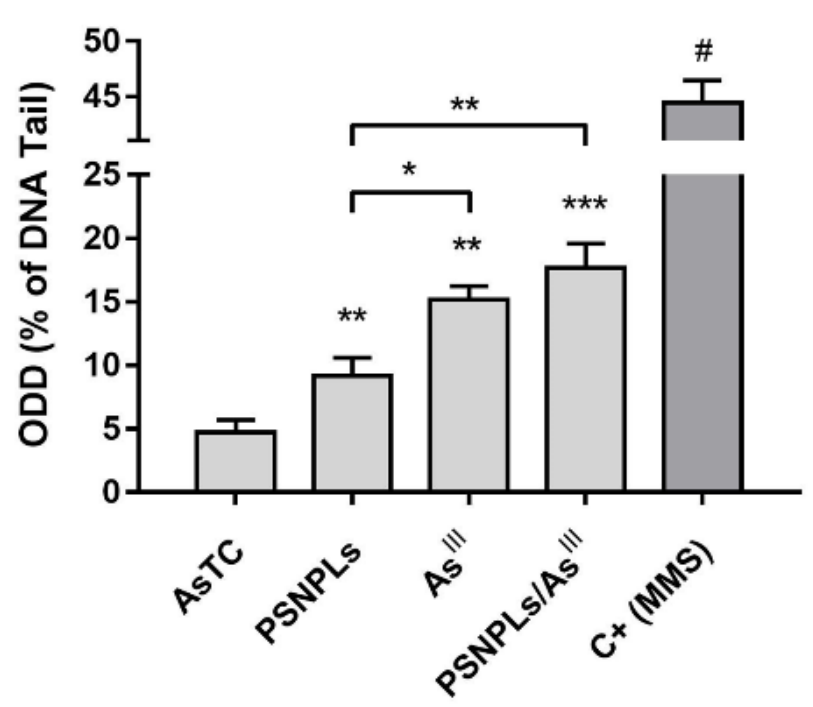

Figure 4

DNA damage induced by the long-term (co)exposure to AsIll and PSNPLs measured by the comet assay.

(A) Total and (B) oxidative DNA damage levels after a 12-week exposure of AsTC to $25 \mu \mathrm{g} / \mathrm{mL}$ PSNPLs, 2 $\mu \mathrm{M}$ AsIII and the combination of both PSNPLs/AsIII treatments. Methyl methanesulfonate (MMS; 200 $\mu \mathrm{M})$ was used as a positive control. Data are presented as mean \pm SEM analyzed by one-way ANOVA

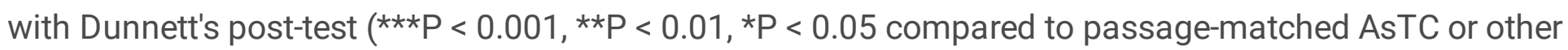
conditions as indicated; $\# \mathrm{P}<0.001$ when compared to all tested conditions). 

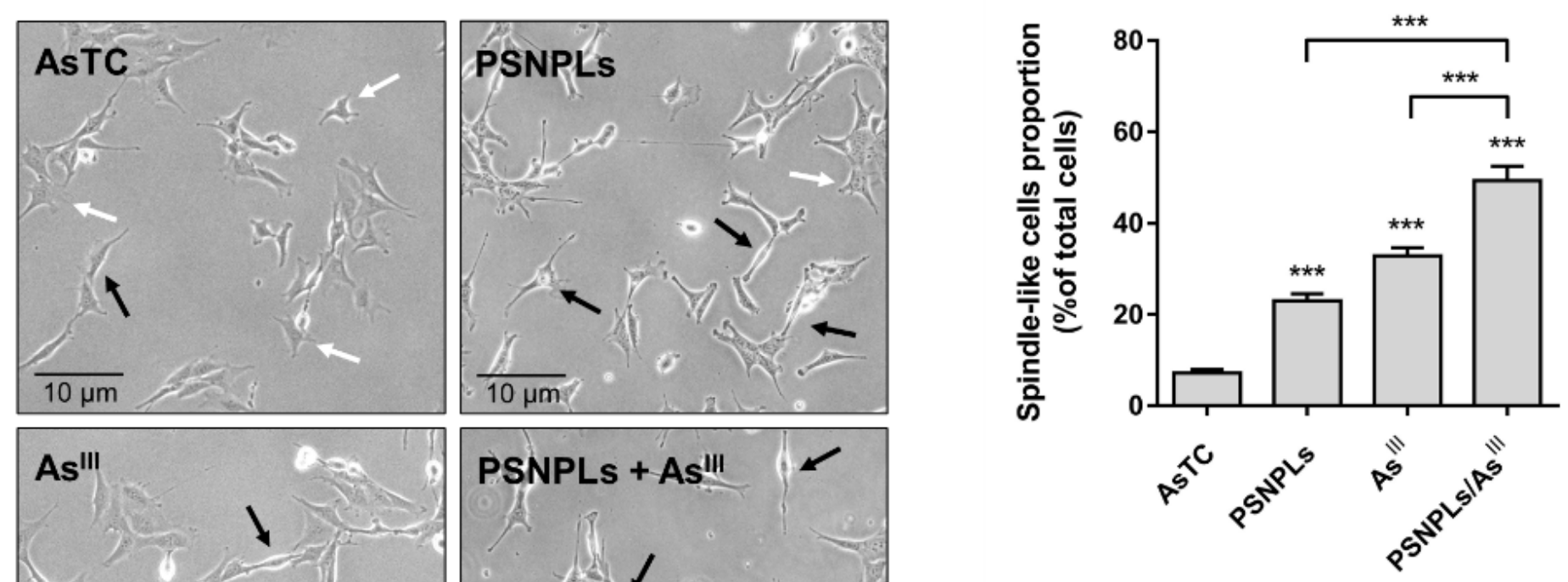

\section{Figure 5}

Chronically (co)exposed AsTC morphology evaluation. (A) Representative images of non-transformed MEF cells, non-exposed AsTC, and AsTC after the chronic exposure to $25 \mu \mathrm{g} / \mathrm{mL}$ PSNPLs, $2 \mu \mathrm{M}$ AsIll, and the combination of both PSNPLs/AsIll. Arrows point to cells with flattened stellate shape (white) or to spindle-like cells (black). (B) Proportion of spindle-like cells quantified in 5 different fields for each tested condition. Data are presented as mean \pm SEM analyzed by one-way ANOVA with Dunnett's post-test ( $* \star * P$ $<0.001$, compared to passage-matched AsTC or other conditions as indicated)

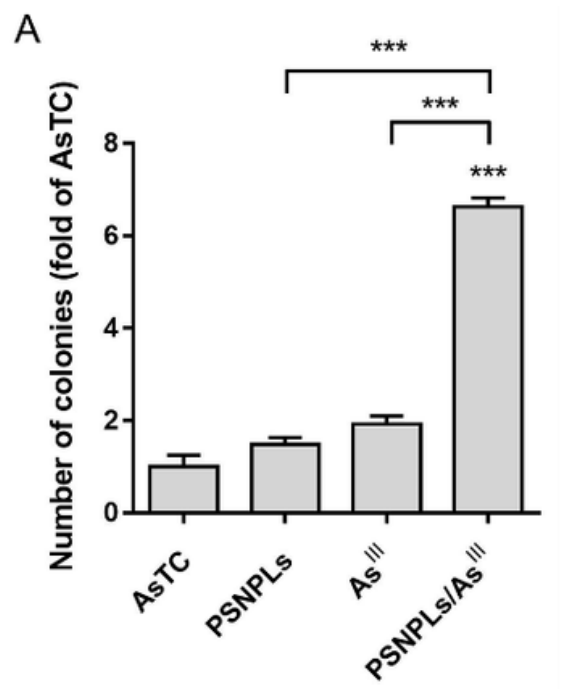

B

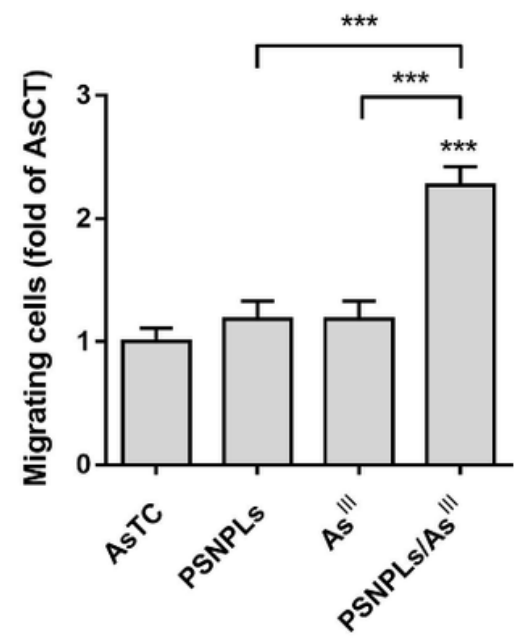

C

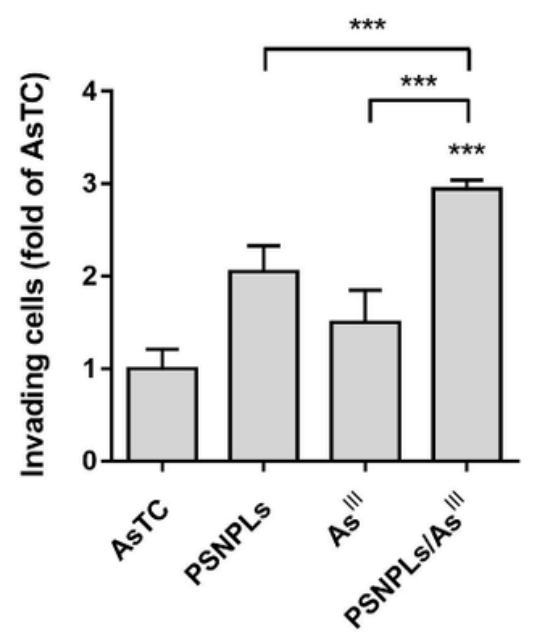

Figure 6 
Determination of the in vitro transformed phenotype after the chronic (co)exposure. (A) Quantitative data derived from the number of colonies formed in the anchorage-independent growth assay. (B) Proportion of cells able to translocate to the basolateral side of the transwell in the migration, and (C) invasion assays. Data are represented as fold of the mean comparing exposed AsTC with the passage-matched AsTC. Error bars represent SEM. \#P $<0.001$ when compared to all conditions tested by the one-way ANOVA with Dunnett's post-test.

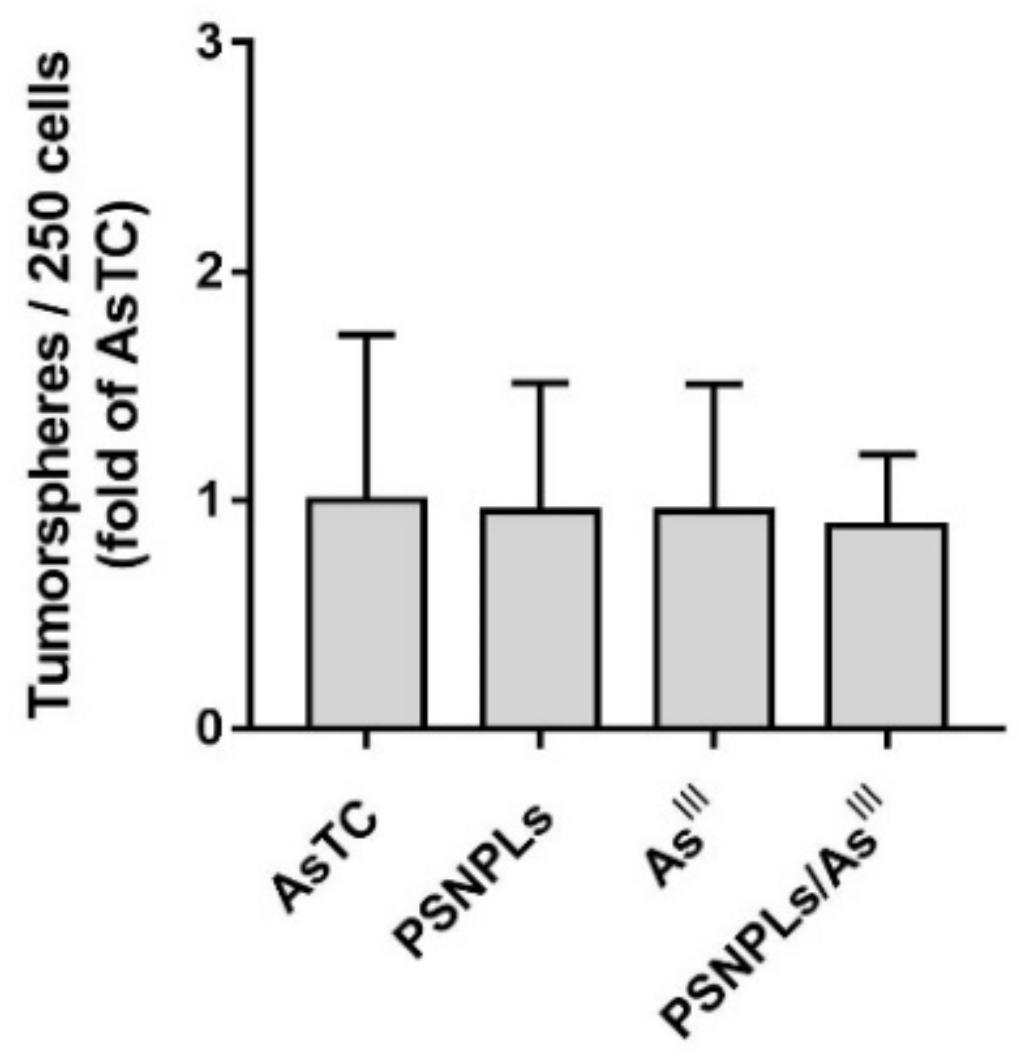

Figure 7

Evaluation of the proportion of stem-like cells in chronically (co)exposed cultures. Number of tumorspheres formed for every 250 AsTC seeded under tumorsphere-inducing conditions. Data are represented as fold of the mean comparing exposed AsTC with the passage-matched AsTC. Error bars represent SEM. 